\title{
„Mathematik ist unerlässlich, um die UN-Ziele für nachhaltige Entwicklung zu erreichen"
}

\author{
Thomas Vogt im Gespräch mit Christiane Rousseau
}

\begin{abstract}
Die Popularisierung von Mathematik liegt Christiane Rousseau seit langem am Herzen: Sie hat Mathematics on Planet Earth 2013 (MPE2013) initiiert, ein von der UNESCO unterstütztes Projekt. Nach dem großen Erfolg von MPE2013 hat sie sich für einen Internationalen Tag der Mathematik, ausgerufen von der UNESCO, eingesetzt und dieses Ziel im vergangenen Jahr erreicht. In diesem Jahr hat am 14. März der erste Internationale Tag der Mathematik stattgefunden, Anlass für die Mitteilungen, kurz vor dem IDM mit der engagierten Mathematikerin zu sprechen.
\end{abstract}

Erinnern Sie sich noch daran, wessen Idee es war, sich für einen internationalen Tag der Mathematik bei der UNESCO zu bewerben?

Bei der International Mathematical Union (IMU) hatten wir darüber diskutiert, welche Rolle die IMU bei der Popularisierung der Mathematik spielen könnte. Die Antwort war nicht offensichtlich, da die Popularisierung oft auf lokaler Ebene und in den jeweiligen Landessprachen erfolgt. Wie könnte also die Zusammenarbeit gefördert werden? Seit 2015 bin ich Mitglied des wissenschaftlichen Beirats des International Basic Science Program (IBSP) der UNESCO, und ich hatte das Dossier des Internationalen Tags des Lichts gesehen. Da kam mir die Idee eines Internationalen Tags der Mathematik, kurz IDM. Ein IDM schien mir die Möglichkeit zu bieten, die Reichweite zu erhöhen. Ich hatte Mathematics of Planet Earth 2013 (MPE2013) organisiert und dafür damals die Schirmherrschaft der UNESCO erhalten. Zudem hatte ich einige Erfahrung mit der UNESCO. Ich erwähnte die Idee gegenüber Jean-Paul Ngome von der UNESCO und sein Enthusiasmus überzeugte mich, die Idee voranzutreiben.

Welche (wichtigen) Schritte mussten unternommen werden, um den IDM umzusetzen?

Der erste Schritt bestand darin, die Idee eines IDM zu diskutieren und ein Datum dafür zu finden, das noch kein offizieller internationaler Gedenktag war. Zu diesem Zweck konsultierte die IMU ihre Mitglieder über ihre anhängenden Organisationen. Den Mitgliedern wurden drei Fragen gestellt, von denen die erste lautete, ob sie einen IDM befürworten, die bis auf eine Ausnahme mit Ja beantwortet wurde. Die zweite Frage betraf Vorschläge für das Datum und die dritte, welche Zeiträume im Jahr für die Feier eines IDM nicht geeignet wären. Etwa 15 Länder schlugen den Pi-Tag vor, alle anderen Vorschläge kamen nur einmal. Wir hatten Glück, denn der 14. März war ein passendes Datum für alle Länder der Welt.
Nachdem das Datum gewählt war, ging es im nächsten Schritt darum, ein Dossier zu erstellen: Dazu gehörte die Beschreibung des Projekts, seiner Ziele und seiner praktischen Umsetzung. Insbesondere über letztere musste nachgedacht werden, da die IMU nur über begrenzte Ressourcen verfügt und sie die Durchführung einer neuen großen Aktivität nicht einfach übernehmen konnte. Als die Generalversammlung der IMU das Prinzip eines Internationalen Tags der Mathematik genehmigte, wurde auch beschlossen, dass die praktische Durchführung ausgelagert werden müsste. Die IMU ernannte einen Lenkungskreis, dessen Vorsitz ich innehabe und der den Auftrag hat, alle praktischen Aspekte der Umsetzung zu koordinieren, einschließlich der Website als Hauptknotenpunkt.

\section{Und wie haben Sie den IDM bei der UNESCO durchbe-} kommen?

Das bringt uns zum dritten Schritt. Er bestand darin, das Projekt bei der UNESCO vorzuschlagen und durchzusetzen. Die Mitglieder der UNESCO sind die Länder und nur ein Mitgliedsstaat kann die Ausrufung eines internationalen Tags beantragen. Seitens IMU baten wir nun alle beteiligten Organisationen um ihre Hilfe, d. h. ihre ständige Vertretung bei der UNESCO davon zu überzeugen, die Proklamation eines IDM vorzuschlagen. Ich traf mich persönlich mit mehreren Delegationen bei der UNESCO: Alle waren im Prinzip einverstanden, aber lange wollte keine den Vorschlag machen. Parallel dazu nahm ich Kontakt zu vielen internationalen, regionalen und nationalen Organisationen auf, um den großen Rückhalt für das Projekt in der ganzen Welt deutlich zu machen. Professor Saliou Touré, Präsident der Mathematischen Gesellschaft der Elfenbeinküste, schaffte den Durchbruch und überzeugte die UNESCO-Delegation seines Landes, den IDM vorzuschlagen. Sofort schlossen sich 37 weitere Delegationen dem Antrag an. Der Antrag wurde dann im Oktober 2018 auf die Tagesordnung der 205. Sitzung des Exekutivrats 
der UNESCO gesetzt, dort befürwortet und dann im November 2019 auf der 40. Hauptkonferenz der UNESCO abschließend genehmigt.

Was waren wichtige Meilensteine auf dem Weg zum ersten IDM?

Damit der IDM erfolgreich sein kann, ist es wichtig, dass er weltweit gefeiert wird. Aus diesem Grund wurde viel Mühe darauf verwendet, das Projekt bekannt zu machen und Partnerorganisationen zu identifizieren, die die Initiative unterstützen und bewerben würden (z. B. durch ihre Newsletter) und die Aktivitäten auf nationaler und lokaler Ebene durchführen würden. Bei diesen Partnerorganisationen handelte es sich hauptsächlich um mathematische Gesellschaften, mathematischnaturwissenschaftliche Institute, einige Universitäten und einige wenige Museen. Inzwischen haben wir unseren eigenen IDM-Newsletter, der alle unsere Abonnenten über die neuen Entwicklungen informiert.

Die Entwicklung des Logos erfolgte im Rahmen eines internationalen Wettbewerbs.

Es gab auch eine Ausschreibung für die IDM-Website. IMAGINARY hat hierfür den Zuschlag bekommen, hat die Website erstellt und hostet sie nun. Im Großen und Ganzen war das internationale Netzwerk von IMAGINARY äußerst hilfreich bei der weltweiten Bekanntmachung des IDM.

Was war während des Prozesses hilfreich und was gab es für Probleme?

Der schwierigste Schritt auf dem Weg zur Ausrufung eines internationalen Tags der Mathematik bestand wie gesagt darin, ein Land zu finden, dessen Delegation bei der UNESCO die Proklamation vorschlagen würde. Auf der Generalkonferenz war die Unterstützung vieler afrikanischer Länder unter der Führung der Elfenbeinküste und Ghanas sehr hilfreich. Das ist übrigens kein Zufall: Feste sind Teil der afrikanischen Kultur und in den letzten Jahren haben viele afrikanische Länder eine Kultur der mathematischen und naturwissenschaftlichen Feste in der Öffentlichkeit entwickelt. Auch feierten mehrere afrikanische Länder bereits den Pi-Tag.

Wie ging es dann weiter?

Jeder internationale Tag muss bei der UNESCO offiziell eingeführt werden. Wir haben viel Energie aufgewendet, um ein schönes Programm für einen offiziellen Start im UNESCO-Hauptquartier vorzubereiten, der am 13. März 2020 stattfinden sollte, da der 14. März 2020 ein Samstag war. Das Programm umfasste zwei Vorträge von Fieldsmedaillengewinnern und Unterhaltungsaktivitäten für die breite Öffentlichkeit. Mehrere Schulen wollten mit ihren Schülerinnen und Schülern zur Veranstaltung kommen. Es hätte sogar ein gleichzeitiges Start-Event des Next Einstein Forums 2020 (NEF) in Nairobi, Kenia, als Plenarveranstaltung gegeben. Das NEF trifft sich alle zwei Jahre und bringt etwa 2000 Menschen aus ganz Afrika und

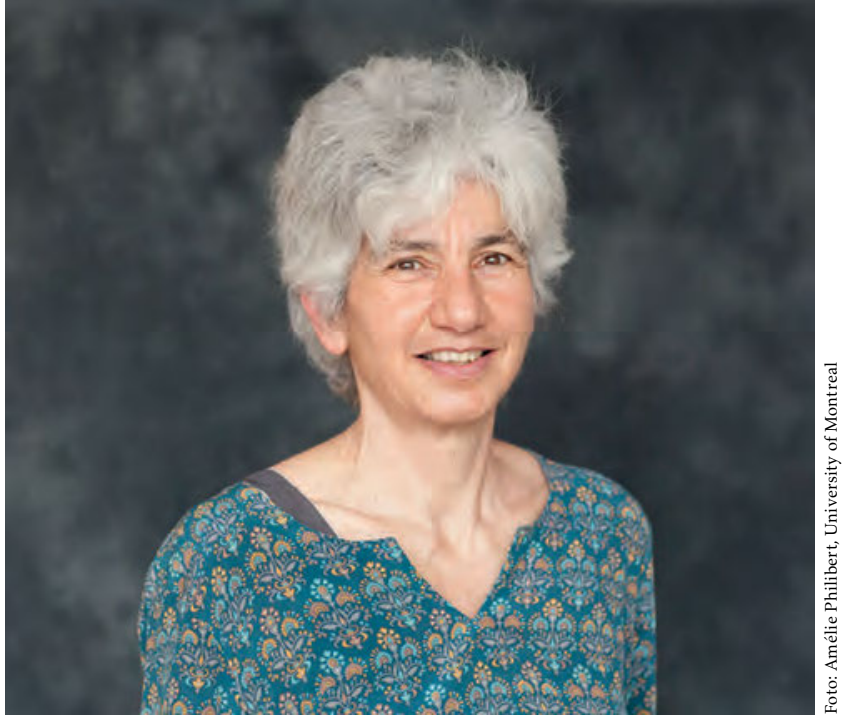

Christiane Rousseau

allen wissenschaftlichen Disziplinen zusammen. Die Startveranstaltung des NEF umfasste zwei Ausstellungen vor Ort sowie Satellitenveranstaltungen. Es war eine große Enttäuschung, als beide Veranstaltungen wegen der COVID-19-Epidemie sehr kurzfristig abgesagt wurden.

\section{Was sind die Ziele des IDM?}

Die Hauptziele des Internationalen Tags der Mathematik sind vielfältig. Ein erstes ist, das Image der Mathematik in der Schule zu verbessern und die Mathematik als eine Disziplin zu präsentieren, die Spaß machen und interessant sein kann, letztendlich mit dem Ziel, dass mehr Kinder MINT-Karrieren in Betracht ziehen.

Ein IDM ist eine gute Gelegenheit, Lobbyarbeit für die Disziplin zu machen, also in der Öffentlichkeit, in den Medien und bei Entscheidungsträgern für die Bedeutung der Mathematik in der Bildung zu werben.

Bei der Formulierung weiterer Ziele haben wir uns oft auf die Ziele der Vereinten Nationen für nachhaltige Entwicklung (SDG1-17, tinyurl.com/ph4ntgn) bezogen. Ein besonderer Schwerpunkt liegt dabei auf dem Aufbau von Kapazitäten in der mathematischen und naturwissenschaftlichen Bildung, mit besonderem Augenmerk auf Kinder und Jugendliche in Entwicklungsländern (SDG4). Denn die Mathematik ist ein Instrument für die Entwicklung und den Übergang zu einer leistungsfähigen Wirtschaft (SDG9). Der IDM hat auch die Aufgabe, Frauen und Mädchen in Mathematik zu befähigen (SDG 5 ) und so zur Gleichstellung der Geschlechter beizutragen. Der IDM könnte vielen Ländern in der Welt helfen, die Grundlagenforschung in Mathematik als Keimzelle für Durchbrüche in Technologie und Management zu begreifen (SDG8). Der IDM ist auch eine Gelegenheit, die Rolle der Mathematik in der menschlichen Zivilisation, einschließlich ihrer Wirtschafts-, Finanz-, Gesundheits- und Verkehrssysteme, der Telekommunikation und beim Bemühen um Wohlergehen $\left(\mathrm{SDG}_{3}\right)$ hervorzuheben sowie ihre Rolle bei der Bekämpfung von Katastrophen, Epidemien, neu auftretenden Krankheiten und invasiven Arten (SDG11) aufzuzeigen. 

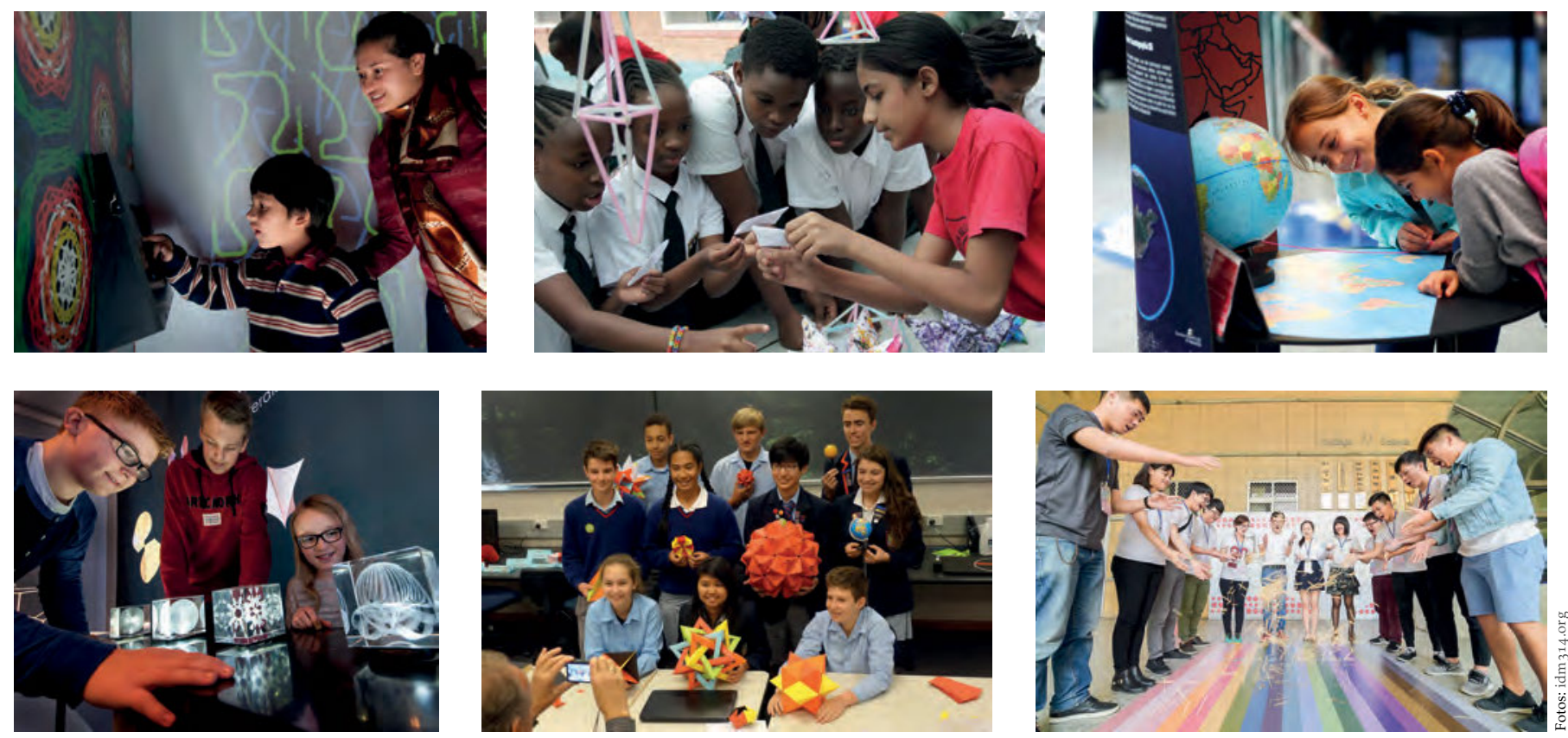

Lokal organisierte Veranstaltungen in Buenos Aires, Kigali, London, Trondheim, Christchurch und Taichung (v. 1.n. r. und v. o. n. u.)

Was war die Idee dabei „Mathematik ist überall“ als Motto für $2020 \mathrm{zu}$ wählen?

Die Wahl des Mottos für das Jahr 2020 folgte dem gleichen Prinzip wie die Wahl des Datums des IDM. Die Mitglieder der IMU wurden konsultiert und um Vorschläge gebeten. „Mathematik ist überall“ wurde von Jaime Carvalho e Silva aus Portugal, dem ehemaligen Generalsekretär des ICMI, vorgeschlagen und vom IDM-Lenkungskreis ausgewählt. Die Breite des Themas machte es zum idealen Motto für 2020. Es enthält die Anwendungen der Mathematik in Naturwissenschaft und Technik, die Rolle der Mathematik beim Verständnis und bei der Bewältigung der planetaren Herausforderungen und die Ziele der Agenda 2030 der Vereinten Nationen für nachhaltige Entwicklung. Das Thema fördert die Kreativität und kann sogar aus dem Blickwinkel der Künste und der $\mathrm{Mu}-$ sik betrachtet werden. Um den Menschen zu helfen, sich das Thema anzueignen, haben wir folgende Unterthemen aufgezählt:

- Mathematik steckt in Wissenschaft und Technik

- Mathematik ist Teil der Zivilisation.

- Mathematik ist unerlässlich, um die UN-Ziele für nachhaltige Entwicklung zu erreichen.

- Mathematik steckt in allem, was Sie tun, und

- Erzählen Sie mir von einer Aktivität oder einem Bereich, und ich werde Ihnen sagen, wo die Mathematik steckt.

Da das letzte Unterthema den Eindruck erwecken könnte, dass Mathematik von außen kommt, sollte das vorletzte Unterthema jeden befähigen, Mathematik um sich herum zu finden.

Last but not least eignet sich das Motto 2020 hervorragend für Politik und Medien.
Wie ist die Reaktion aus der Mathematik und den verschiedenen Ländern?

Unserem Eindruck zufolge ist die Reaktion enthusiastisch. Wir, die IMU, erhalten viel Lob und Glückwünsche für das IDM-Projekt. Aber unser größter Lohn sind die IDM-Projekte auf der ganzen Welt in über 110 Ländern, übrigens auch in Ländern, die wir ursprünglich nicht kontaktiert haben, wie Albanien, Armenien, Bolivien, Bosnien und Herzegowina, Gambia, Irak, Kasachstan, Liberia, Namibia, Malaysia, Panama und Thailand usw. Nepal wollte sogar nicht bis 2020 warten, um den IDM zu feiern und hat bereits 2019 Feierlichkeiten organisiert, für die es eigene Banner gedruckt hat. Wir erhalten viele Anfragen für die Übersetzung des Logos, das inzwischen in 22 Sprachen existiert.

\section{Glauben Sie, dass der IDM2020 ein Erfolg wird? Wenn ja, nennen Sie bitte Beispiele.}

Unserer Meinung nach ist der IDM2O20 ein großer Erfolg. In vielen Ländern nehmen die Menschen die Veranstaltung an, sie verbreitet sich im ganzen Land. Beispielsweise waren in Portugal 139 Feierlichkeiten angekündigt, 126 in der Türkei, 84 in Italien, 42 in Kolumbien, 32 in Kroatien, 20 in Deutschland, 19 in Südafrika, auf den Philippinen 15, in Malaysia und Bosnien und Herzegowina je zehn sowie weitere in mindestens 26 Ländern Afrikas.

Aber die wirkliche Bewährungsprobe sind die kommenden Jahre: Wir hoffen, dass die Zahl und Vielfalt der Feierlichkeiten von Jahr zu Jahr wächst, statt eine einmalige Sache zu sein. Als Beispiel für die unterschiedlichste Art von Feierlichkeiten möchte ich Portugal anführen: Viele 


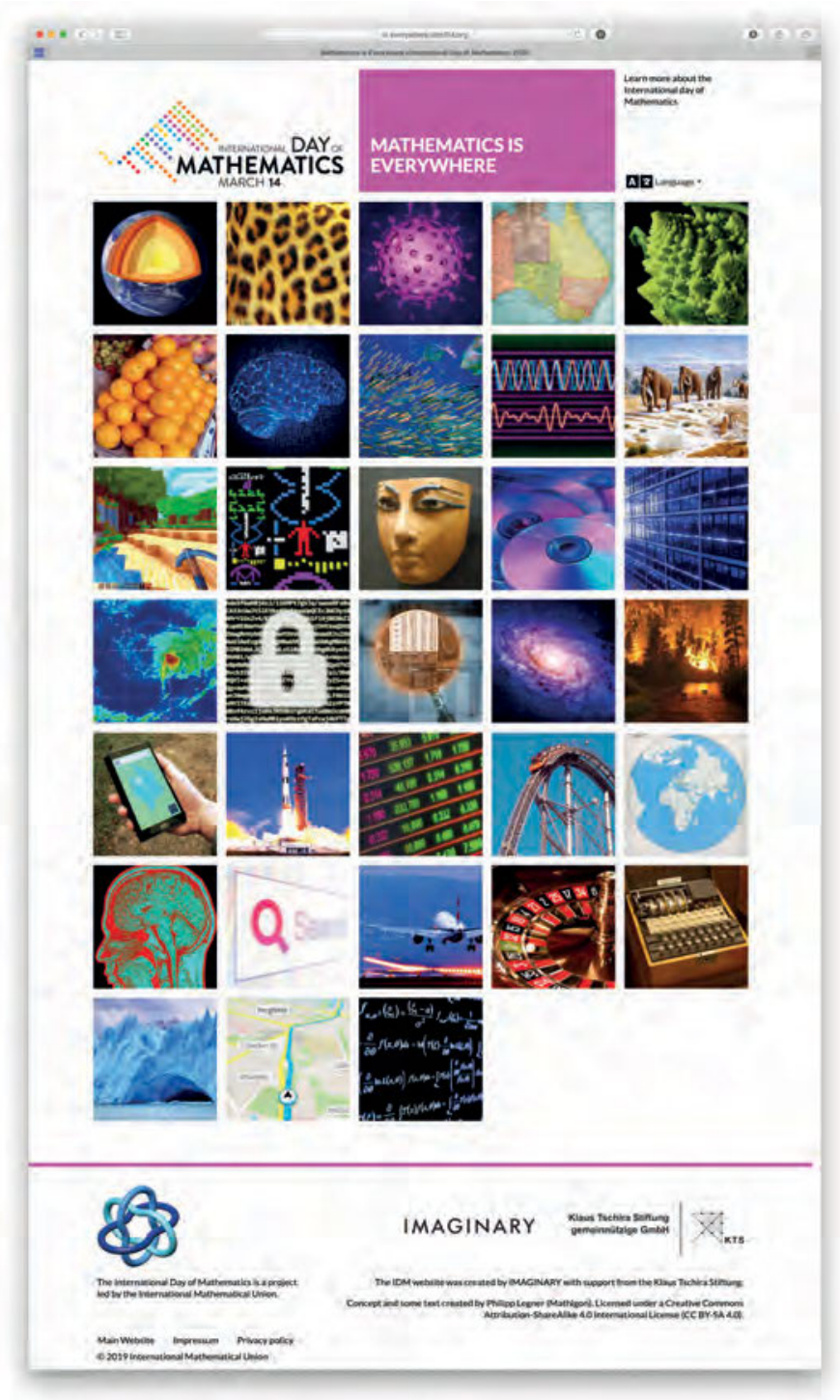

Veranstaltungen fordern die portugiesischen Schülerinnen und Schüler heraus, sich eine Welt ohne Mathematik vorzustellen. Ein lokaler Radiosender organisiert ein „PiFestival“ mit Preisen für die besten Gedichte und Musik, inspiriert von der Zahl $\pi$.

Was wird das Motto des IDM2021 sein?

Es liegt in der Verantwortung des IDM-Lenkungskreises, das Motto des IDM2O21 zu wählen. Wir werden den gleichen Weg wie beim IDM2O20 einschlagen, d. h. wir wer- den zunächst die IMU-Mitglieder über ihre Mitgliedsorganisationen konsultieren. Zudem werden wir die anderen Vorschläge, die wir im Jahr 2020 erhalten haben, in die Diskussionen einbeziehen. Ein wichtiges Kriterium bei der Wahl des Themas ist, dass es für Aktivitäten auf allen Ebenen, also in Schulen, aber auch auf Universitätsebene sowie in der Öffentlichkeit und den Medien geeignet ist.

Das Interview wurde kurz vor dem IDM202o geführt. Übersetzung aus dem Englischen von Marc Oprisiu, DMV-Medienbüro.

Christiane Rousseau lehrt seit 1979 an der Universität Montreal, seit 1991 auf einer vollen Professur. Sie war 2008/2009 Direktorin des Centre de Recherches Mathématiques an der Universität Montreal. Von 2002 bis 2004 war Christiane Rousseau Präsidentin der Kanadischen Mathematischen Gesellschaft, von 2011 bis 2014 Vizepräsidentin der International Mathematical Union.

Thomas Vogt und Marc Oprisiu, Medienbüro Mathematik, FU Berlin, Institut für Mathematik, Königin-Luise-Straße 24-26, 14195 Berlin medienbuero@mathematik.de 